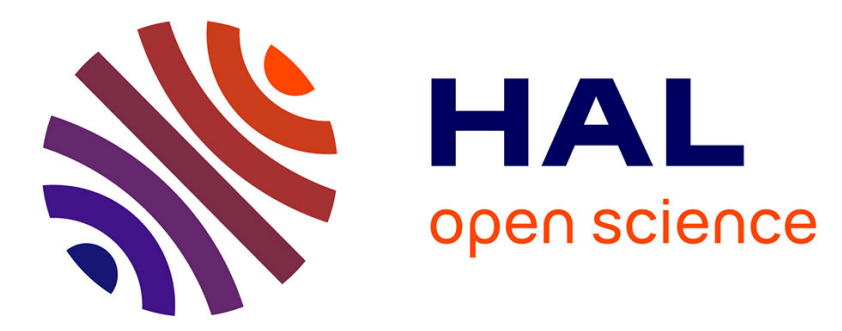

\title{
An algal photoenzyme converts fatty acids to hydrocarbons
}

\author{
Damien Sorigué, Bertrand Legeret, Stéphan Cuiné, Stephanie Blangy, Solène \\ Y. Moulin, Emmanuelle Billon, Pierre Richaud, Sabine Brugière, Yohann \\ Couté, Didier Nurizzo, et al.
}

\section{To cite this version:}

Damien Sorigué, Bertrand Legeret, Stéphan Cuiné, Stephanie Blangy, Solène Y. Moulin, et al.. An algal photoenzyme converts fatty acids to hydrocarbons. Science, 2017, 357 (6354), pp.903 - 907. 10.1126/science.aan6349 . hal-01775093

\section{HAL Id: hal-01775093 \\ https://hal-amu.archives-ouvertes.fr/hal-01775093}

Submitted on 24 Apr 2018

HAL is a multi-disciplinary open access archive for the deposit and dissemination of scientific research documents, whether they are published or not. The documents may come from teaching and research institutions in France or abroad, or from public or private research centers.
L'archive ouverte pluridisciplinaire HAL, est destinée au dépôt et à la diffusion de documents scientifiques de niveau recherche, publiés ou non, émanant des établissements d'enseignement et de recherche français ou étrangers, des laboratoires publics ou privés. 


\title{
An algal photoenzyme converts fatty acids to hydrocarbons
}

1. Damien Sorigué ${ }^{1}$, Bertrand Légeret ${ }^{1}$, Stéphan Cuiné $^{1}$, Stéphanie Blangy ${ }^{1}$, Solène Moulin', Emmanuelle Billon', Pierre Richaud ${ }^{1}$, Sabine Brugière ${ }^{2}$, Yohann Couté $^{2}$, Didier Nurizzo ${ }^{3}$, Pavel Müller ${ }^{4}$, Klaus Brettel $^{4}$, David Pignol ${ }^{5}$, Pascal Arnoux $^{5}$, Yonghua Li-Beisson ${ }^{1}$, Gilles Peltier ${ }^{1}$, Fred Beisson ${ }^{1},{ }^{*}$

1. Biosciences and Biotechnologies Institute of Aix-Marseille (BIAM), Commissariat à I'Energie Atomique et aux Energies Alternatives (CEA), CNRS and Aix-Marseille University, UMR 7265 LB3M, CEA Cadarache, F-13108, Saint-Paul-lez-Durance, France.

2. University Grenoble Alpes, CEA and INSERM, BIG-BGE, F-38000, Grenoble, France.

3. European Synchrotron Radiation Facility, 71 Avenue des Martyrs, CS 40220, F38043 Grenoble, France.

4. Institute for Integrative Biology of the Cell (I2BC), CEA, CNRS, University Paris-Sud, University Paris-Saclay, F-91198, Gif-sur-Yvette cedex, France.

5. BIAM, CEA, CNRS and Aix-Marseille University, UMR 7265 LBC, CEA Cadarache, F-13108, Saint-Paul-lez-Durance, France.

•Corresponding author. Email: frederic.beisson@cea.fr

\begin{abstract}
Although many organisms capture or respond to sunlight, few enzymes are known to be driven by light. Among these are DNA photolyases and the photosynthetic reaction centers. Here, we show that the microalga Chlorella variabilis NC64A harbors a photoenzyme that acts in lipid metabolism. This enzyme belongs to an algae-specific clade of the glucose-methanol-choline oxidoreductase family and catalyzes the decarboxylation of free fatty acids to n-alkanes or -alkenes in response to blue light. Crystal structure of the protein reveals a fatty acid-binding site in a hydrophobic tunnel leading to the light-capturing flavin adenine dinucleotide (FAD) cofactor. The decarboxylation is initiated through electron abstraction from the fatty acid by the photoexcited FAD with a quantum yield $>80 \%$. This photoenzyme, which we name fatty acid photodecarboxylase, may be useful in light-driven, bio-based production of hydrocarbons.
\end{abstract}

In both photosynthetic and nonphotosynthetic organisms, light is involved in biological processes such as growth, development, metabolism, and circadian entrainment (14). In most cases, the effects of light on cell physiology are mediated by photoactive proteins, which include light-sensitive ion channels and pumps, photoreceptors, photosynthetic antenna proteins, and light-dependent enzymes (5). Among the latter, two different types can be distinguished. Light-activated enzymes require only a flash of light to become active, whereas photoenzymes require a continuous flux of photons to remain catalytically active- - their activity is driven by light.

Photoenzymes are a rare type of catalyst. In nature, two families of proteins bearing a light-driven activity are DNA photolyases, which are involved in repairing ultraviolet (UV) damage in DNA in many organisms, from bacteria to plants and animals (6-8), and light-dependent protochlorophyllide reductases, which catalyze chlorophyll synthesis (9). Chlorophyll f synthase might be a third type of photoenzyme, but the 
light-driven nature of the reaction has not been established (10). The multimeric complexes that form the basis of oxygenic photosynthesis-plastocyanin:ferredoxin oxidoreductase (often referred to as photosystem I) (11) and water:plastoquinone oxidoreductase (photosytem II) (12), as well as related proteins in nonoxygenic photosynthetic bacteria - may also be classified as photoenzymes (5).

Photoenzymes are useful tools for research and biotechnology. For example, lightcontrolled catalysis allows the study of rapid enzymatic processes in real time (13). And, the use of a photoexcited cofactor to design an enzyme with a different catalytic function $(14,15)$ as well as the development of optogenetics $(16,17)$ expands the scope of possible applications of enzymes driven by light.

Some microalgae, including Chlorella variabilis NC64A and Chlamydomonas reinhardtii $137 \mathrm{C}$, have the capacity to convert long-chain fatty acids to alkanes or alkenes in a process dependent on light (18). Because no homologs to known hydrocarbon-forming enzymes have been found in the genomes of $C$. variabilis or $C$. reinhardtii, we hypothesized that microalgae harbor a different type of alkane synthase. Conversion of microalgal fatty acids to hydrocarbons has been mostly studied in Botryococcus braunii (19). Here, we identify an alkane synthase in the green microalgae $C$. variabilis and show that it is a photoenzyme belonging to an algaespecific subfamily of proteins that has been conserved throughout algal evolution.

\section{Results}

\section{Identification of a microalgal alkane synthase}

In order to identify the enzyme responsible for hydrocarbon synthesis in microalgae, we undertook an approach of enzyme purification in $C$. variabilis. Following the observation that $C$. variabilis cells can convert exogenous fatty acids into alkanes or alkenes (18), an activity assay for a hydrocarbon-forming enzyme was set up (fig. S1A). This discontinuous assay was sensitive enough to allow a partial purification of the protein responsible for the alkane-forming activity (fig. S1B). Proteomic comparison of three partially purified fractions resulted in a list of 10 common proteins, nine of which were homologous to well-characterized proteins (Fig. 1A). The most represented protein, and also the only one whose function was uncertain, was a member of the glucose-methanol-choline (GMC) oxidoreductases, a family of flavoproteins comprising a wide range of oxidases and dehydrogenases (20). A full-length cDNA coding for the $C$. variabilis GMC oxidoreductase was cloned and found to encode a polypeptide of 654 amino acids ( 69 kDa) (Fig. 1B). Expression in Escherichia coli of the corresponding codon-optimized synthetic gene (fig. S2) resulted in the production of $\mathrm{C} 13$ to $\mathrm{C} 17$ alkanes or alkenes (Fig. 1C), which demonstrated that this protein was indeed a hydrocarbon-forming enzyme. 


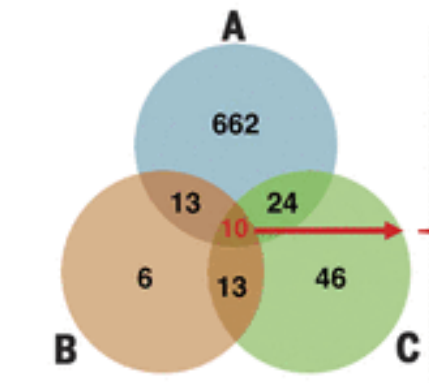

Number of proteins detected

in each of 3 purifications $(A, B, C)$

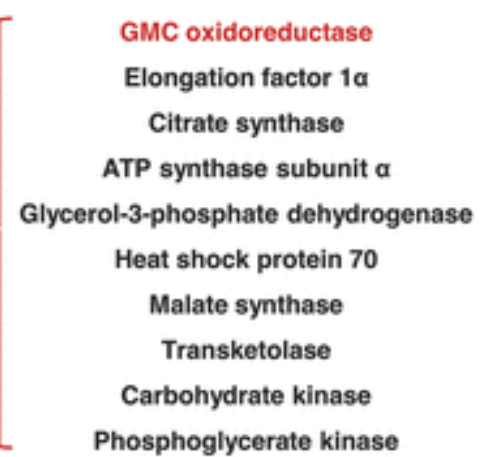

Phosphoglycerate kinase

B
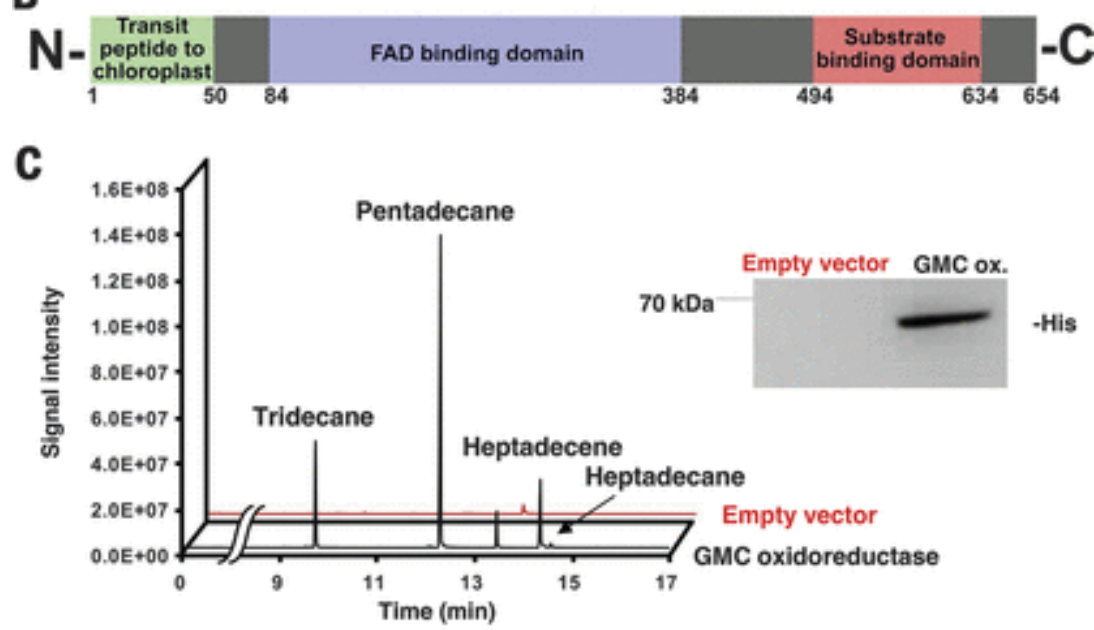

Fig. 1 Identification of an alkane synthase in C. variabilis.

(A) Number of proteins found after the final step in three independent purifications and list of the 10 proteins in common found by means of proteomic analysis. Proteins are ranked by decreasing spectral counts. (B) Predicted domains of the $C$. variabilis $G M C$ oxidoreductase. (C) Chromatogram of the hydrocarbons produced (left) and immunoblot of the total proteins (right) in an $E$. coli strain expressing the $C$. variabilis GMC oxidoreductase (alkane synthase).

\section{Characterization of the fatty acid decarboxylase activity}

To further characterize the activity of the $C$. variabilis GMC oxidoreductase, we expressed the recombinant protein in $E$. coli and purified it. Mass spectrometry analysis revealed the presence of a flavin adenine dinucleotide (FAD) cofactor (fig. S3), as observed in other characterized GMC oxidoreductases (20). Characterization of the hydrocarbon-forming activity of the recombinant protein showed that this activity was optimum around $\mathrm{pH} 8.5$ and decreased sharply when temperature rose above $35^{\circ} \mathrm{C}$ (fig. S3). No activity could be detected by using either a fatty acid methyl ester or a fatty aldehyde as substrate. In order to determine whether the reaction catalyzed by the alkane synthase was an actual decarboxylation of fatty acid, palmitic acid labeled with ${ }^{13} \mathrm{C}$ on the carboxylic group was used as substrate. Upon incubation of the substrate with the $C$. variabilis alkane synthase, production of ${ }^{13} \mathrm{CO}_{2}$ was detected at more than 20 times its natural abundance (Fig. 2A), thus indicating that the enzyme was a fatty acid decarboxylase. 

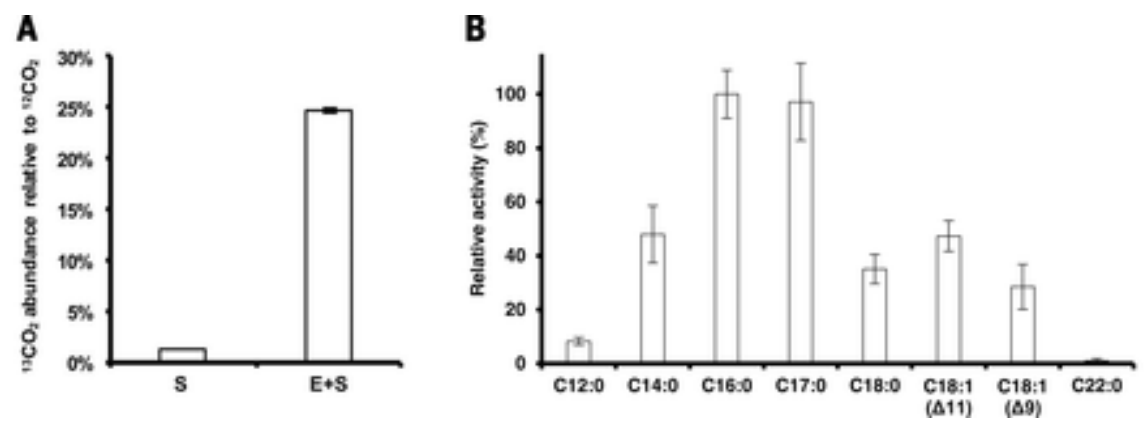

Fig.2 Fatty acid decarboxylase activity of the C. variabilis alkane synthase.

(A) Relative quantification of ${ }^{13} \mathrm{CO}_{2}$ released upon incubation of $1-{ }^{13} \mathrm{C}$-palmitate with the purified recombinant enzyme. S, substrate; $E$, enzyme. (B) Relative activity of the purified recombinant enzyme on various free fatty acid substrates. Mean $\pm S D$ ( $n=3$ repeats).

The purified enzyme was then tested on a wide range of free fatty acid substrates ( $\mathrm{C} 12$ to C22) (Fig. 2B). All of them were converted into $\mathrm{C}_{n-1}$ alkanes or alkenes, with a higher efficiency for C16-C17 chains. Saturated fatty acids were converted to alkanes, not alkenes, which showed that the $C$. variabilis fatty acid decarboxylase did not introduce a terminal double bond in the carbon chain, unlike the two fatty acid decarboxylases identified in bacteria $(21,22)$. We also identified a GMC oxidoreductase from $C$. reinhardtii with features and substrate specificities similar to its $C$. variabilis homolog (fig. S4).

\section{Light-dependence of the $\mathrm{C}$. variabilis alkane synthase}

In algal cells, alkane formation requires light (18). The fact that the $C$. variabilis alkane synthase contains a chromophore, together with the observation that the purified enzyme does not require any additional cofactor to be active, prompted us to check the light dependency of the enzyme activity. We first observed that when the $E$. coli strain expressing the $C$. variabilis alkane synthase was cultivated under complete dark and never exposed to light, no hydrocarbon was produced (fig. S3G). Measurement of the activity of the purified $C$. variabilis enzyme at different wavelengths of visible light showed further that the alkane synthase was active in the presence of blue light (400 to $520 \mathrm{~nm}$ ) (Fig. 3A). The action spectrum correlated with FAD absorbance, thus suggesting that FAD is the light-capturing part of the protein. However, these observations did not discriminate between a light-activated enzyme and a photoenzyme. The activity of the purified enzyme on $1-{ }^{13} \mathrm{C}-$ labeled palmitic acid was thus measured under different light conditions by monitoring the release of ${ }^{13} \mathrm{CO}_{2}$. We observed that ${ }^{13} \mathrm{CO}_{2}$ production started immediately upon illumination with blue light and stopped immediately after a shift to dark or red light (Fig. 3B). This demonstrated that the $C$. variabilis alkane synthase is a photoenzyme because it requires a continuous flux of (blue) photons for its activity. The same feature was also noted on the purified $C$. reinhardtii GMC oxidoreductase (fig. S4D). Alkane formation increased with light intensity (Fig. 3C). Using palmitic acid as substrate, we estimated the turnover number of the $C$. variabilis GMC oxidoreductase at $0.86 \pm 0.13 \mathrm{~s}^{-1}$ under a fluence rate of $2000 \mu \mathrm{mol}$ photons $\mathrm{m}^{-2} \mathrm{~s}^{-1}$ white light. The photoexcitation rate (supplementary materials, materials and methods) in this experiment was $\sim 0.9 \mathrm{~s}^{-1}$, indicating efficient photocatalysis (quantum yield $>80 \%$ ). On the basis of these results, we propose the name fatty acid photodecarboxylase (FAP) for the $C$. variabilis GMC oxidoreductase and its $C$. reinhardtiihomolog. 

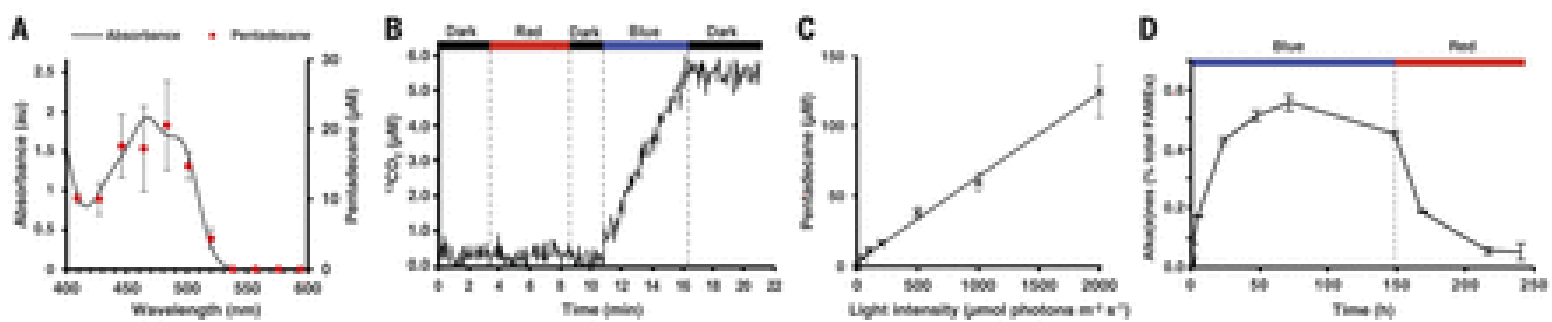

Fig.3 Light dependency of the C. variabilis alkane synthase.

(A) Absorption spectrum and action spectrum of the purified enzyme. Mean $\pm S D(n=3$ repeats). The position of the flavin absorption maximum in the enzyme (467 nm) deviates from typical values (445 to $450 \mathrm{~nm}$ for most flavoproteins and free flavins). (B) Activity of the purified enzyme under successive light conditions. Activity on $1-{ }^{13} \mathrm{C}$-palmitate was monitored via release of ${ }^{13} \mathrm{CO}_{2}$ by using membrane inlet mass spectrometry. (C) Dependence of the activity of the purified enzyme on white light intensity. Mean $\pm S D(n=3$ repeats). (D) Variation of total hydrocarbons in $C$. reinhardtii cells grown in a photobioreactor under blue and then red light. Mean $\pm S D$ ( $n=3$ repeats). FAMEs, fatty acid methyl esters.

The blue light dependency of the FAP prompted us to investigate the effect of the quality of light on the formation of hydrocarbons in algal cultures. When $C$. reinhardtii cultures were grown in photobioreactors under successively blue $(<500 \mathrm{~nm})$ and red $(>600 \mathrm{~nm}$ ) lights, alkane or alkene formation was correlated with the presence of blue light (Fig. 3D). This experiment thus showed that the light dependency of the FAP observed in vitro was relevant to living cells and likely to be related to its function in the cell.

\section{Three-dimensional structure of the FAP}

In order to gain insights into the structural basis of the light-dependent fatty acid decarboxylase activity of the $C$. variabilis FAP, we determined its three-dimensional (3D) structure in complex with a palmitate substrate (table S1). A crystal form diffracting to 3.15-Å resolution was obtained. Although the crystal was twinned, we were able to solve its structure via molecular replacement by using domains from two proteins of the GMC oxidoreductase family with known 3D structures (PDB codes 2JBV and 4YNT). The overall architecture of the FAP was similar to other GMC oxidoreductases, with a typical two-domain fold (Fig. 4A). The first domain, mostly shaped by N-terminal residues, stabilizes the FAD cofactor, whereas the second domain participates in substrate/product trafficking, with loops from both domains shielding the active site from the solvent and holding together both domains.
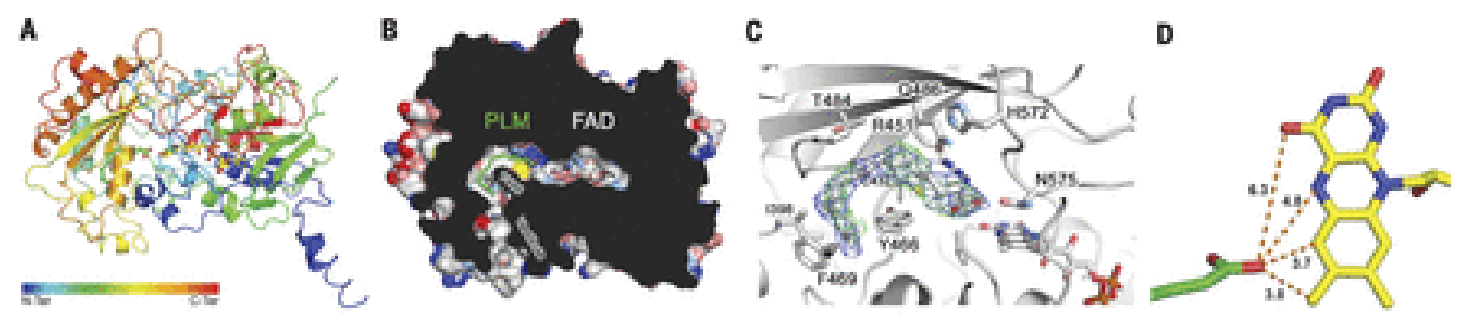

Fig.4 Structural features of the C. variabilis FAP.

(A) Overall architecture of the enzyme in complex with FAD and palmitate (PLM). The structure is represented as an illustration colored from blue to red from the $N$ to the $C$ terminus, with the $F A D$ and palmitic acid represented in stick. The long $N$-terminal helix is projected toward a noncrystallographic symmetry-related molecule that is not represented here for clarity. (B) 
Slice through the surface representation of the fatty acid photodecarboxylase. For clarity, small cavities in the interior of the enzyme are not shown. (C) Details of the palmitic acid-binding site with the side chains of residues within $4 \AA$ of the substrate shown in stick. The omit map electron density associated to palmitic acid is also shown and contoured at $0.5 \sigma\left(2 F_{o}-F_{c} ;\right.$ blue) and $2 \sigma\left(F_{o}-F_{c} ;\right.$ green). Single-letter abbreviations for the amino acid residues are as follows: A, Ala; C, Cys; D, Asp; E, Glu; F, Phe; G, Gly; H, His; I, Ile; K, Lys; L, Leu; M, Met; N, Asn; P, Pro; Q, Gln; R, Arg; S, Ser; T, Thr; V, Val; W, Trp; and Y, Tyr. (D) Distances (angstroms) between palmitic acid and FAD.

The 3D structure of the FAP revealed a narrow hydrophobic tunnel, open to the solvent on one end and leading to the tricyclic ring of FAD on the other end (Fig. 4B). Electron density in this tunnel indicates the stabilization of a palmitate substrate that wraps around the side chain of $Y 466$, with the carboxylate moiety interacting with the side chains of residues R451, C432, and Q486 (Fig. 4C) and pointing toward the FAD (shortest distance $3.7 \AA$ ) (Fig. 4D). The lower enzyme activity for medium-chain fatty acids (Fig. 2B) may be caused by a less efficient stabilization of short hydrophobic chains in the tunnel (especially around Y466). A multiple sequence alignment of GMC oxidoreductases of the FAP subgroup showed that most of the amino acids of the hydrophobic tunnel present in the vicinity of the FAD or lining the entry site of palmitate (vestibule) were conserved (fig. S5).

\section{Role of FAD in the FAP photocycle}

To elucidate the role of the FAD cofactor in the decarboxylation of fatty acids by FAP, we performed time-resolved optical spectroscopy measurements on the purified recombinant enzyme. The fluorescence kinetics (Fig. 5A) show that the substrate quenches the singlet excited state ${ }^{1} \mathrm{FAD}^{*}$, shortening its lifetime from $\mathrm{T} \sim 5$ ns (typical time constant for unquenched flavins (23) to $\mathrm{T} \sim 300$ ps. FAD-based reaction intermediates and kinetics were resolved by means of transient absorption spectroscopy on nanosecond to millisecond time scales. We presumed that the $300-$ ps kinetics might reflect electron transfer (ET) from the substrate to ${ }^{1} F A D^{*}$. Indeed, at $376 \mathrm{~nm}$ (where FAD*-absorbs more strongly than FAD) (Fig. 5B) an instantaneous (within the instrumental time resolution of $10 \mathrm{~ns}$ ) absorbance increase was observed (Fig. 5C, black trace), whereas at $488 \mathrm{~nm}$ (where FAD*- absorbs less than FAD) (Fig. 5B), an initial bleaching occurred (Fig. 5C, blue trace). Measurements at other wavelengths confirmed the rapid formation of FAD"- (fig. S6, A and B). At most wavelengths studied, the initial absorbance changes decayed with $\mathrm{T} \sim 100 \mathrm{~ns}$ (Fig. $5 \mathrm{C}$ and fig. S6A), indicating reoxidation of $\mathrm{FAD}^{--}$to $\mathrm{FAD}$. At $515 \mathrm{~nm}$, however, the absorbance rose in $\sim 100 \mathrm{~ns}$ (Fig. 5C, green trace) and decayed with $\mathrm{T} \sim 4 \mathrm{~ms}$ (Fig. 5C, inset). Because the slope of the FAD absorption spectrum in FAP is steep at $515 \mathrm{~nm}$ (Fig. 5B), we considered the possibility that after reoxidation of $F_{A D}{ }^{\circ-}$, the FAD spectrum was red-shifted because of a change in its environment resulting from the decarboxylation of the substrate. Spectral analysis of the 4-ms phase (fig. S6C) confirmed such a red-shift.
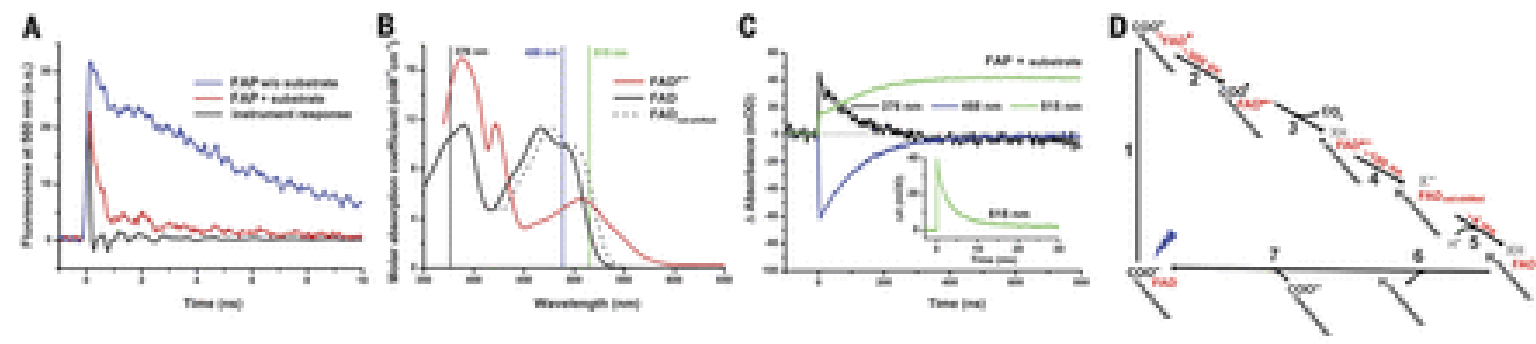
Fig.5 Characterization of C. variabilis FAP by means of time-resolved spectroscopy.

(A) Time-resolved fluorescence of FAP in the presence or absence of palmitic acid. (B) Absorption spectra of FAD"- [in glucose oxidase (31); red line] and oxidized FAD in FAP (solid black line), scaled assuming a molar absorption coefficient of $11300 \mathrm{M}^{-1} \mathrm{~cm}^{-1}$ for the maximum of the absorption band in the blue (32). A red-shifted blue band of FAD in FAP (dotted black line) is shown to demonstrate the effect on the absorption at $515 \mathrm{~nm}$. (C) Examples of transient absorption changes of FAP in the presence of excess substrate. Complementary data are shown in fig. S6. (D) Suggested model of the FAP photocycle. Directly observed intermediates and kinetics are shown in red.

In the absence of substrate, flash-induced absorbance changes show the formation of the FAD triplet state ${ }^{3} \mathrm{FAD}^{*}$ (fig. S6, D and E), which is consistent with an intersystem crossing yield in the order of $70 \%$ for unquenched singlet excited flavins (23) and decay of ${ }^{3} \mathrm{FAD}^{*}$ to ground state $\mathrm{FAD}$ in $\sim 80 \mu \mathrm{s}$. In the presence of substrate, formation of ${ }^{3} \mathrm{FAD}^{*}$ should be largely outcompeted by ET from the substrate to ${ }^{1} \mathrm{FAD}^{*}$ in $~ 300 \mathrm{ps}$ (fig. S6F), which is consistent with a high quantum yield of photocatalysis.

\section{FAP clade in phylogenetic tree of GMC oxidoreductases}

All members of the GMC oxidoreductase family characterized so far are oxidases or dehydrogenases (mostly acting on sugars), except some plant lyases that catalyze breaking of a C-C bond (24) and the FAP, which is also a lyase. We analyzed the phylogenetic relationships of 50 prokaryotic and eukaryotic members of the GMC oxidoreductase family, including 20 proteins with demonstrated biochemical activities. $C$. variabilis and $C$. reinhardtii FAP did not group with the plant clade but instead belonged to a separate clade that contained only sequences from algae (Fig. 6 , table S2, and fig. S7). This algal-specific clade included micro- and macroalgae and algae of primary or secondary endosymbiotic origin. Among the 22 algal species with sequenced genomes, 18 had a GMC oxidoreductase in the FAP clade. The four other algae (Ostreococcus and Micromonas species, from the same basal Mamiellophyceae class of green algae) lacked a predicted GMC oxidoreductase. Algal species with several GMC oxidoreductases were all of secondary endosymbiotic origin (such as Nannochloropsis, Phaeodactylum, or Emiliana). Thus, the algal clade of GMC oxidoreductases is likely to be a group with a conserved FAP function. 


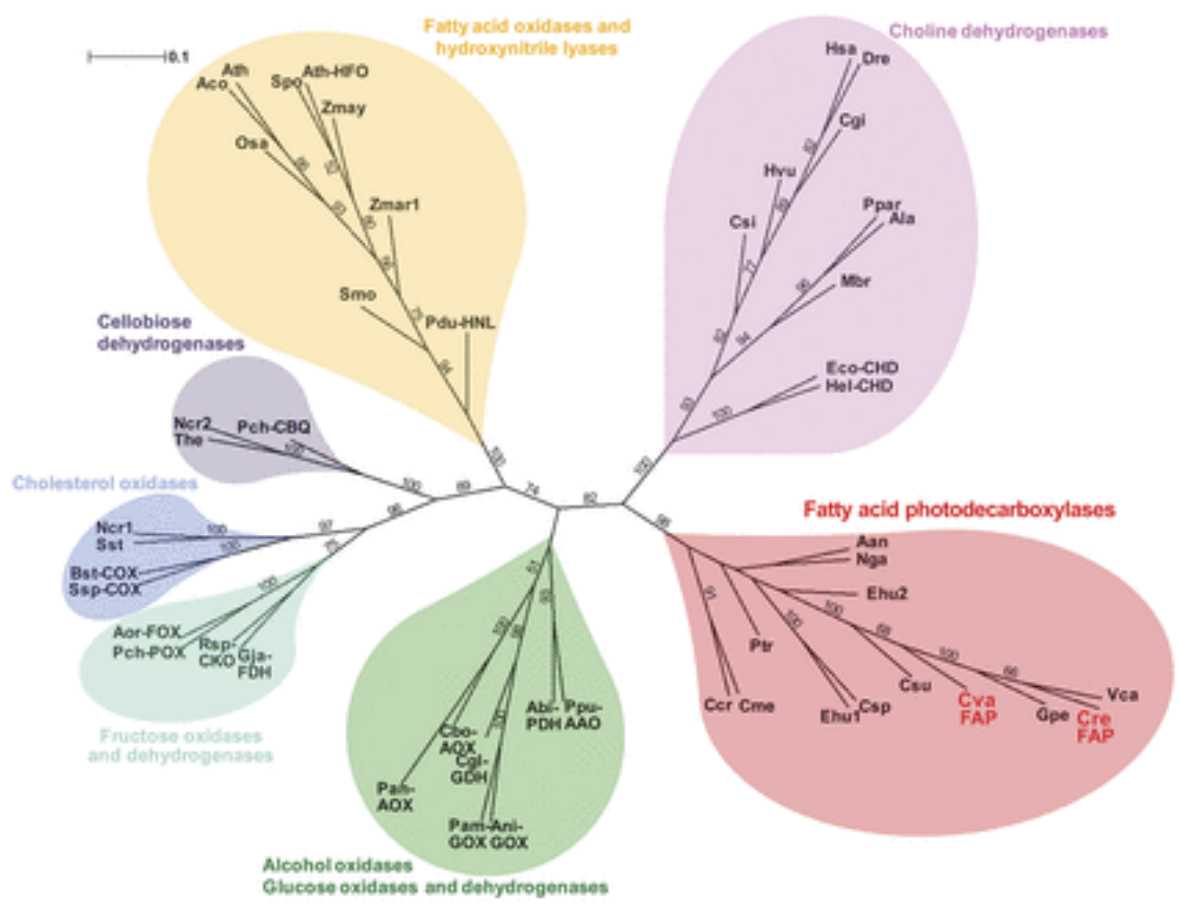

Fig.6 Unrooted phylogenetic tree of the GMC oxidoreductase family.

The neighbor joining method was used. Species are abbreviated to three letters (one for the genus and two for the species epithet) (table S2). Proteins with demonstrated biochemical activity are indicated by three additional capital letters: $A A O$, aryl alcohol oxidase; $A O X$, alcohol oxidase; FAP, fatty acid photodecarboxylase; $C B Q$, cellobiose dehydrogenase; $C H D$, choline dehydrogenase; COX, cholesterol oxidase; $C K O$, compound $K$ oxidase; $F D H$, fructose dehydrogenase; FOX, fructose oxidase; GDH, glucose dehydrogenase; GOX, glucose oxidase; HFO, hydroxy fatty acid oxidase (genetic evidence only); HNL, hydroxynitrile lyase; and POX, pyranose oxidase.

\section{Discussion}

Here, we identified a microalgal alkane synthase as a member of the GMC oxidoreductase family and a photoenzyme. Known enzymes with hydrocarbon-forming activity include oxidoreductases and polyketide synthases in cyanobacteria $(25,26)$, fatty acid desaturase-like proteins CER1 and CER3 in plants (27), cytochrome P450s in the bacterium Jeotgalicoccus sp. ATTC8456 and in insects $(21,28)$, and nonheme diiron oxidoreductases in the bacterium Pseudomonas aeruginosa(22). Algal GMC oxidoreductases now join that group but have the added feature of being light-driven. Photoenzymes are an ancestral type of enzyme and may be rare because evolutionary selection has not favored light-driven catalysis (29). Given the diversity of proteins able to perform synthesis of alkanes, light activation required by the chemistry seems unlikely. We therefore speculate that the light-driven nature of the microalgal alkane synthases is related to their function within the cell. The FAP function likely appeared after primary endosymbiosis, was kept during secondary endosymbiosis, but was lost in plants. Conservation of the FAP function in algae throughout evolution suggests a biological function specific to this group of organisms.

Because $C$. reinhardtii and $C$. variabilis FAP are active on various fatty acids in vitro, the formation in $C$. reinhardtii and $C$. variabilis cells of a predominant species of hydrocarbon (7-heptadecene) (18) may be due to the channeling of the precursor (cisvaccenic acid) to the FAP. This result suggests that the $C$. reinhardtii and $C$. 
variabilis alkane synthases interact with a yet-to-be-identified lipolytic enzyme that releases cis-vaccenic acid from a lipid or cofactor.

We propose a model of the FAP photocycle (Fig. 5D) that combines observed FADbased intermediates and kinetics (in red) with inferred (not directly observed) corresponding changes in the substrate. The FAD in FAP is first photoexcited (step 1). The singlet excited state ${ }^{1} \mathrm{FAD}^{*}$ abstracts an electron from the fatty acid in $\sim 300$ ps (step 2). The fatty acid radical decarboxylates yielding an alkyl radical [step 3; not directly observed but analogous to the photo-Kolbe reaction (30)]. FAD ${ }^{-1}$ transfers the electron back to the resulting alkyl radical in $100 \mathrm{~ns}$ (step 4). Because of the extremely strong proton affinity of alkyl anions, we suppose that the ET is coupled to proton transfer from a proton donor $\mathrm{XH}$ (one of the neighboring amino acid residues or a water molecule). Alternatively, $\mathrm{XH}$ may transfer a hydrogen atom to the primary alkyl radical, followed by ET from FAD ${ }^{--}$to $X^{\bullet}$ in $\sim 100$ ns. We speculate that the resulting negative charge of $\mathrm{X}^{-}$may cause a red shift of the FAD absorption band in the visible. The FAD band shifts back to the blue in $\sim 4 \mathrm{~ms}$ (step 5), possibly because of reprotonation of $\mathrm{X}^{-}$. The alkane is released (step 6), and a new substrate is bound (step 7). Judging from the crystal structure, we considered the best candidates for $\mathrm{XH}$ would be cysteine $\mathrm{C} 432$ and tyrosine Y466 (Fig. 4C). In order to test our hypotheses about the involvement of a proton donor $\mathrm{XH}$ in the catalytic reaction, we have mutated these two residues to alanine, which should not be able to function as a proton or hydrogen atom donor. Although 50 to $60 \%$ of purified wild-type enzyme contained FAD, none of the mutant proteins bound the FAD cofactor. Thus, the hypothesis could not be tested.

Our identification of a photoenzyme acting on lipids shows that light-driven catalysis is not restricted to the processes of light capture and use or to the repair of UV damages in DNA. This discovery should thus provide an incentive to reinvestigate the activity of some enzymes involved in metabolic or signaling pathways regulated by light.

\section{References and Notes}

\section{C. L. Thompson, A. Sancar}

Photolyase/cryptochrome blue-light photoreceptors use photon energy to repair DNA and reset the circadian clock.

Oncogene 21, 9043-9056 (2002).doi:10.1038/sj.onc.1205958pmid:12483519

\section{K. Greenham, C. R. McClung}

Integrating circadian dynamics with physiological processes in plants. Nat. Rev. Genet. 16, 598-610 (2015). doi:10.1038/nrg3976pmid:26370901

3. T. Yoshii, C. Hermann-

Luibl, C. Kistenpfennig, B. Schmid, K. Tomioka, C. Helfrich-Förster

Cryptochrome-dependent and -independent circadian entrainment circuits in Drosophila. J.

Neurosci. 35, 6131-6141(2015). doi:10.1523/JNEUROSCI.0070-

15.2015pmid:25878285 
4 K. K. Fuller, J. J. Loros, J. C. Dunlap

Fungal photobiology: Visible light as a signal for stress, space and time.

Curr. Genet. 61, 275-288 (2015). doi:10.1007/s00294-014-0451-0pmid:25323429

5 L. O. Björn, Photoactive proteins, in Photobiology, The Science of Light and Life, L. O. Björn, Ed. (Springer, ed. 3, 2015) pp. 139-150.

6 K. Brettel, M. Byrdin

Reaction mechanisms of DNA photolyase.

Curr. Opin. Struct. Biol. 20, 693-701(2010).

doi:10.1016/j.sbi.2010.07.003pmid:20705454

7 D. Zhong

Electron transfer mechanisms of DNA repair by photolyase.

Annu. Rev. Phys. Chem. 66, 691-715(2015).

doi:10.1146/annurev-physchem-040513-103631pmid:25830375

8 A. Sancar

Mechanism of DNA repair by photolyase and excision nuclease (Nobel Lecture). Angew. Chem. Int. Ed. 55, 8502-8527 (2016).

doi:10.1002/anie.201601524pmid:27337655

9 M. Gabruk, B. Mysliwa-Kurdziel

Light-dependent protochlorophyllide oxidoreductase: Phylogeny, regulation, and catalytic properties.

Biochemistry54, 5255-5262 (2015).

doi:10.1021/acs.biochem.5b00704pmid:26230427

10 M. Y. Ho, G. Shen, D. P. Canniffe, C. Zhao, D. A. Bryant

Light-dependent chlorophyll $f$ synthase is a highly divergent paralog of PsbA of photosystemll.

Science 353, aaf9178 (2016).doi:10.1126/science.aaf9178pmid:27386923

11 A. N. Melkozernov, J. Barber, R. E. Blankenship

Light harvesting in photosystem I supercomplexes

Biochemistry 45, 331-345 (2006). doi:10.1021/bi051932opmid:16401064

12 G. Renger, T. Renger

Photosystem II: The machinery of photosynthetic water splitting.

Photosynth. Res.98, 53-80 (2008). doi:10.1007/s11120-008-9345-7pmid:18830685

13 D. Zhong 
Ultrafast catalytic processes in enzymes.

Curr. Opin. Chem. Biol. 11, 174-181 (2007).

doi:10.1016/j.cbpa.2007.02.034pmid:17353141

14 M. A. Emmanuel, N. R. Greenberg, D. G. Oblinsky, T. K. Hyster

Accessing non-natural reactivity by irradiating nicotinamide-dependent enzymes with light.

Nature 540, 414-417 (2016).doi:10.1038/nature20569pmid:27974767

15 U. T. Bornscheuer

Chemical biology: A radical change in enzyme catalysis.

Nature 540, 345-346(2016). doi:10.1038/540345apmid:27974776

16 D. M. Shcherbakova, A. A. Shemetov, A. A. Kaberniuk, V. V. Verkhusha

Natural photoreceptors as a source of fluorescent proteins, biosensors, and optogenetic tools.

Annu. Rev. Biochem. 84, 519-550(2015).

doi:10.1146/annurev-biochem-060614-034411pmid:25706899

17 K. Zhang, B. Cui

Optogenetic control of intracellular signaling pathways.

Trends Biotechnol. 33, 92-100(2015).

doi:10.1016/j.tibtech.2014.11.007pmid:25529484

18 D. Sorigué, B. Légeret, S. Cuiné, P. Morales, B. Mirabella, G. Guédeney, Y. LiBeisson, R. Jetter, G. Peltier,F. Beisson

Microalgae synthesize hydrocarbons from long-chain fatty acids via a light-dependent pathway.

Plant Physiol. 171, 2393-2405 (2016). pmid:27288359

19 M. Dennis, P. E. Kolattukudy

A cobalt-porphyrin enzyme converts a fatty aldehyde to a hydrocarbon and $\mathrm{CO}$.

Proc. Natl. Acad. Sci. U.S.A. 89, 5306-5310 (1992).

doi:10.1073/pnas.89.12.5306pmid:1608940

20 D. R. Cavener

GMC oxidoreductases. A newly defined family of homologous proteins with diverse catalytic activities.

J. Mol. Biol. 223, 811-814 (1992). doi:10.1016/0022-2836(92)90992-Spmid:1542121

21 M. A. Rude, T. S. Baron, S. Brubaker, M. Alibhai, S. B. Del Cardayre, A. Schirmer Terminal olefin (1-alkene) biosynthesis by a novel p450 fatty acid decarboxylase from Jeotgalicoccus species.

Appl. Environ. Microbiol. 77, 1718-1727 (2011).

doi:10.1128/AEM.02580-10pmid:21216900

22 Z. Rui, X. Li, X. Zhu, J. Liu, B. Domigan, I. Barr, J. H. D. Cate, W. Zhang 
Microbial biosynthesis of medium-chain 1-alkenes by a nonheme iron oxidase.

Proc. Natl. Acad. Sci. U.S.A. 111, 18237-18242 (2014).

doi:10.1073/pnas.1419701112pmid:25489112

23 P. F. Heelis

The photophysical and photochemical properties of flavins (isoalloxazines).

Chem. Soc. Rev.11, 15-39 (1982). doi:10.1039/cs9821100015

24 I. Dreveny, K. Gruber, A. Glieder, A. Thompson, C. Kratky

The hydroxynitrile lyase from almond: A lyase that looks like an oxidoreductase.

Structure 9, 803-815 (2001). doi:10.1016/S0969-2126(01)00639-6pmid:11566130

25 A. Schirmer, M. A. Rude, X. Li, E. Popova, S. B. del Cardayre

Microbial biosynthesis of alkanes.

Science329, 559-562 (2010). doi:10.1126/science.1187936pmid:20671186

26 D. Mendez-Perez, M. B. Begemann, B. F. Pfleger

Modular synthase-encoding gene involved in a-olefin biosynthesis in Synechococcus sp. strain PCC 7002.

Appl. Environ. Microbiol. 77, 4264-4267 (2011).

doi:10.1128/AEM.00467-11pmid:21531827

27 A. Bernard, F. Domergue, S. Pascal, R. Jetter, C. Renne, J.-D. Faure, R.

P. Haslam, J. A. Napier, R. Lessire,J. Joubès

Reconstitution of plant alkane biosynthesis in yeast demonstrates that ArabidopsisECERIFERUM1 and ECERIFERUM3 are core components of a verylong-chain alkane synthesis complex.Plant

Cell 24, 3106-3118 (2012). doi:10.1105/tpc.112.099796pmid:22773744

28 Y. Qiu, C. Tittiger, C. WickerThomas, G. LeGoff, S. Young, E. Wajnberg,

T. Fricaux, N. Taquet, G. J.Blomquist, R. Feyereisen

An insect-specific P450 oxidative decarbonylase for cuticular hydrocarbon biosynthesis.

Proc. Natl. Acad. Sci. U.S.A. 109, 14858-14863 (2012).

doi:10.1073/pnas.1208650109pmid:22927409

29 T. P. Begley

Photoenzymes: A novel class of biological catalysts.

Acc. Chem. Res. 27, 394-401 (1994).doi:10.1021/ar00048a002

30..B. Kraeutler, A. J. Bard

Heterogeneous photocatalytic synthesis of methane from acetic acid - New Kolbe reaction pathway.

J. Am. Chem. Soc. 100, 2239-2240 (1978). doi:10.1021/ja00475a049

31 V. Massey, G. Palmer 
On the existence of spectrally distinct classes of flavoprotein semiquinones. A new method for the quantitative production of flavoprotein semiquinones.

Biochemistry 5, 3181-3189(1966). doi:10.1021/bi00874a016pmid:4382016

32 P. Macheroux,

UV- visible spectroscopy as a tool to study flavoproteins, in Flavoprotein Protocols: Methods in Molecular Biology, S. K. Chapman, G. A. Reid, Eds. (Humana Press, 1999), vol. 131, pp. 1-7.

33 E. H. Harris, The Chlamydomonas Sourcebook: A Comprehensive Guide to Biology and Laboratory Use(Academic Press, 1989).

34 J. Milbradt, A. Kraut, C. Hutterer, E. Sonntag, C. Schmeiser, M. Ferro, S. Wagne, T. Lenac, C. Claus, S.Pinkert, S.T. Hamilton, W.D. Rawlinson, H. Sticht, Y. Couté, M. Marschall

Proteomic analysis of the multimeric nuclear egress complex of human cytomegalovirus.

Mol. Cell. Proteomics 13, 2132-2146(2014).

doi:10.1074/mcp.M113.035782pmid:24969177

35 M.W. Bowler, D. Nurizzo, R. Barrett, A. Beteva, M. Bodin, H. Caserotto, S. Delag enière, F. Dobias, D. Flot, T.Giraud, N. Guichard, M. Guijarro, M. Lentini, G.

A. Leonard, S. McSweeney, M. Oskarsson, W. Schmidt, A.Snigirev, D. von Stetten, J. Surr, O. Svensson, P. Theveneau, C. Mueller-Dieckmann

MASSIF-1: A beamline dedicated to the fully automatic characterization and data collection from crystals of biological macromolecules.

J. Synchrotron Radiat. 22, 1540-1547 (2015).

doi:10.1107/S1600577515016604pmid:26524320

36 P. D. Adams, P. V. Afonine, G. Bunkóczi, V. B. Chen, I. W. Davis, N. Echols, J.

J. Headd, L.-W. Hung, G. J.Kapral, R. W. Grosse-Kunstleve, A. J. McCoy, N.

W. Moriarty, R. Oeffner, R. J. Read, D. C. Richardson, J.

S.Richardson, T.C. Terwilliger, P. H. Zwart

PHENIX: A comprehensive Python-based system for macromolecular structure solution.

Acta Crystallogr. D Biol. Crystallogr. 66, 213-221 (2010).

doi:10.1107/S0907444909052925pmid:20124702

37 F. Long, A. A. Vagin, P. Young, G. N. Murshudov

BALBES: A molecular-replacement pipeline.

Acta Crystallogr. D Biol. Crystallogr. 64, 125-132 (2008).

doi:10.1107/S0907444907050172pmid:18094476

38 P. Emsley, B. Lohkamp, W. G. Scott, K. Cowtan

Features and development of Coot.

Acta Crystallogr. D Biol. Crystallogr. 66, 486-501 (2010).

doi:10.1107/S0907444910007493pmid:20383002 
39 A Espagne, M. Byrdin, A. P. M. Eker, K. Brettel

Very fast product release and catalytic turnover of DNA photolyase.

ChemBioChem 10, 1777-1780 (2009). doi:10.1002/cbic.200900328pmid:19565597

40 M. Byrdin, V. Thiagarajan, S. Villette, A. Espagne, K. Brettel

Use of ruthenium dyes for subnanosecond detector fidelity testing in real time transient absorption.

Rev. Sci. Instrum. 80, 043102 (2009).doi:10.1063/1.3117208pmid:19405646

41 P. Müller, J.-P. Bouly, K. Hitomi, V. Balland, E. D. Getzoff, T. Ritz, K. Brettel

ATP binding turns plant cryptochrome into an efficient natural photoswitch.

Sci. Rep. 4, 5175 (2014).doi:10.1038/srep05175pmid:24898692

42 K. Katoh, K. Misawa, K. Kuma, T. Miyata

MAFFT: A novel method for rapid multiple sequence alignment based on fast Fourier transform.

Nucleic Acids Res. 30, 3059-3066 (2002).doi:10.1093/nar/gkf436pmid:12136088

43 M. Gouy, S. Guindon, O. Gascuel

SeaView version 4: A multiplatform graphical user interface for sequence alignment and phylogenetic tree building.

Mol. Biol. Evol. 27, 221-224 (2010).doi:10.1093/molbev/msp259pmid:19854763

\section{$44 \mathrm{~J}$. Felsenstein}

PHYLIP -Phylogeny inference package (Version 3.2).

Cladistics 5, 164-166 (1989).

45 D. H. Huson, D. C. Richter, C. Rausch, T. Dezulian, M. Franz, R. Rupp

Dendroscope: An interactive viewer for large phylogenetic trees.

BMC Bioinformatics 8, 460 (2007). doi:10.1186/1471-2105-8-460pmid:18034891

46 T. E. Swartz, S. B. Corchnoy, J. M. Christie, J. W. Lewis, I. Szundi, W. R.

Briggs, R. A. Bogomolni

The photocycle of a flavin-binding domain of the blue light photoreceptor phototropin.

J. Biol. Chem. 276,36493-36500 (2001).

doi:10.1074/jbc.M103114200pmid:11443119

\section{Acknowledgments:}

We thank J. Ohlrogge for critical reading of the manuscript. Thanks are also due to $\mathrm{V}$. Cardettini, A. Beyly, I. Té, P. Auroy, A. Beynel, J. Alric, C. Sahut, D. Lemaire, and F. Carrière for technical help or discussions. This work was supported by grants from L'Agence Nationale de la Recherche (ANR) (MUSCA ANR-13-JSV5-0005 to Y.L-B. and $A^{*}$ MIDEX ANR-11-IDEX-0001-02 to G.P.), the European Union (EU) (Sun2Chem project of ERASynBio), the French Infrastructure for Integrated Structural Biology 
(ANR-10-INSB-05-01), the Proteomics French Infrastructure (ANR-10-INBS-08-01), and the HelioBiotec platform funded by the EU, the région Provence-Alpes-Côted'Azur (PACA), the French Ministry of Research, and the CEA. PhD studentships were provided by the région PACA and the CEA (D.S.) and the École Normale Supérieure Paris (S.M.). F.B. conceived the project. P.A., D.N., and D.P. acquired $x$-ray data and resolved the protein structure. S.B. and Y.C. performed the proteomic analysis. P.M. and K.B. performed measurements and analyzed data of time-resolved spectrometry. D.S. executed other experiments and analyzed data with the help of all other authors. F.B., D.S., K.B., P.M., and P.A wrote the paper with contributions from G.P., D.P., Y.C., B.L., P.R., and Y.B.-L. The coding sequence of the $C$. variabilis FAP has been deposited in GenBank under accession no. KY511411. Atomic coordinates and structure factors have been deposited in the Protein Data Bank (PDB) under accession no. $5 \mathrm{NCC}$. All other data needed to evaluate the conclusions in the paper are present in the paper and/or the supplementary materials. D.S., F.B., B.L., S.C., S.B., and G.P. are inventors on patent application EPA 16305583 submitted by CEA and CNRS that covers "New fatty acid decarboxylase and its uses." 